\title{
Echocardiographic Evaluation of Right Ventricular Function in the Immediate Postoperative Period after Major Pulmonary Resections: A Prospective Observational Study
}

\author{
${ }^{1}$ Imran H Bhat, ${ }^{2}$ Alok Kumar, ${ }^{3}$ Balbir Kumar, ${ }^{4}$ Rajarajan Ganesan, ${ }^{5}$ Banashree Mandal, ${ }^{6}$ Vikas Dutta
}

\begin{abstract}
Aim: We aimed to identify the impact through the use of standard and novel echocardiographic parameters, i.e., speckle tracking echocardiography (STE) to evaluate the right and left ventricular (LV) myocardial function in patients who underwent lung resections.
\end{abstract}

Materials and methods: We identified patients that underwent lobectomy or pneumonectomy at our institution in 2016 to 2017. We performed preoperative transthoracic echocardiography (TTE) in each patient and on postoperative days (PODs) 2, 7 , and 30 when available.

Results: Of a total of 26 patients included in the study, 5 underwent pneumonectomy, while the rest underwent lobectomy. Left and right pneumonectomy was performed in 38 and $62 \%$ of the patients respectively. None of the patients had right ventricular (RV) dilation or dysfunction on preoperative echocardiograms. Postoperatively, mean LV ejection fraction (LVEF) was $52( \pm 7.5) \%$. Mean RV strain in immediate postoperative period (day 2 ) was $-15.1 \%$ (reduction of more than $20 \%$ ). None of the patients progressed to RV failure or had mortality. Estimated RV systolic pressure was $41( \pm 20) \mathrm{mm} \mathrm{Hg}$. The differences in RV echocardiographic parameters were significantly different pre- and postsurgery. The RV function decreased significantly on POD 2, which improved slightly thereafter. Extent of resection and side of resection did not make a difference in the RV functions.

Conclusion: After lung resection, patients developed deterioration in RV function that may be reflected by any of the echocardiographic parameters used to assess RV function. Deterioration in RV function is maximum in the immediate postoperative period (day 2), which improves to preoperative level by 4 weeks.

Keywords: Lobectomy, Lung resection, Pneumonectomy, Right ventricle function, Speckle tracking echocardiography.

How to cite this article: Bhat $\mathrm{IH}$, Kumar A, Kumar B, Ganesan R, Mandal B, Dutta V. Echocardiographic Evaluation

\footnotetext{
${ }^{1-4}$ Senior Resident, ${ }^{5}$ Associate Professor, ${ }^{6}$ Assistant Professor

${ }^{1-6}$ Department of Anesthesia and Intensive Care, Postgraduate Institute of Medical Education and Research, Chandigarh, India

Corresponding Author: Alok Kumar, Senior Resident Department of Anesthesia and Intensive Care, Postgraduate Institute of Medical Education and Research, Chandigarh, India Phone: +918146044104, e-mail: docsomi@yahoo.com
}

of Right Ventricular Function in the Immediate Postoperative Period after Major Pulmonary Resections: A Prospective Observational Study. J Perioper Echocardiogr 2017;5(2): 42-48.

Source of support: This work was supported by the Society of Transesophageal Echocardiography.

Conflict of interest: None

\section{INTRODUCTION}

Reduction in the pulmonary vascular bed following major lung surgeries puts the RV at a disadvantage by increasing the afterload. Standard echocardiographic techniques have been used to assess the effects of pulmonary surgery on RV function. ${ }^{1,2}$ Right heart catheterization has been used for the evaluation of right heart function after pneumonectomy with its own limitations and risks. ${ }^{3,4}$ Significant postoperative RV dysfunction manifests on the 2 nd POD. ${ }^{5}$ Tissue Doppler and myocardial deformation have been recently used in clinical echocardiography to accurately evaluate regional and global cardiac function. ${ }^{6-8}$

In this study, we analyze the effect of major pulmonary resections on LV and RV function using conventional as well as a novel echocardiographic technique, i.e., speckle tracking strain. The aim of this study was to analyze the consequence of major pulmonary resections on RV and LV function. We do this by assessing and comparing the global RV and LV functions using tissue Doppler imaging and global longitudinal strain before and after surgery.

\section{MATERIALS AND METHODS}

After approval from the Institute Ethics Committee, patients aged 18 to 60 years scheduled for major pulmonary resection (pneumonectomy, lobectomy) were included in this prospective observational study from Mar 2016 to Mar 2017. Patients with history of myocardial infarction (ischemic heart disease), atrial fibrillation, valvular heart disease, major arrhythmias, heart surgery, heart failure, severe renal failure, and chronic obstructive pulmonary 
disease (COPD) [forced expiratory volume-one second (FEV1)/forced vital capacity (FVC) ratio less than $70 \%]$ were excluded from this study.

All patients were examined a day prior to surgery. Baseline data including preoperative physical status, blood counts, biochemical parameters, preoperative TTE report, and coronary angiography data were noted. Standard anesthesia technique was used for all the patients as per institutional protocol. Thoracic epidural catheter was placed in all patients unless contraindicated. Correct placement of double lumen tube was confirmed by fiberoptic visualization. A comprehensive TTE was performed before induction of general anesthesia with S1-5 phased array probe (GE Medical Systems, Horten, Norway). All measurements were performed online on the same machine by anesthesiologist experienced in TTE blinded to patient's outcome. Patient was started on vasopressor-inotropic support as deemed fit by the treating anesthesiologist to achieve the hemodynamic goal. Weaning and extubation were done by the intensive care unit team.

Echocardiography data: Two-dimensional (2D) echocardiographic examination to measure LV function by

- The LVEF by modified Simpson examination to measure fit by the treating anesthesiologist.

- The LV speckle tracking strain from apical fourchamber, two-chamber, and long-axis views using GE Automated Functional Imaging software.

- Lateral and medial mitral annuli tissue Doppler systolic velocities (S) from apical four-chamber view.

- RV function by Tricuspid valve (TV) inflow velocities (E, A) from apical four-chamber view modified to obtain the best orientation for pulse wave Doppler.

- Tricuspid annular plane systolic excursion (TAPSE) from apical window with best orientation for M-mode.

- $\mathrm{RV}$ fractional area change (RV FAC) from the apical RV focused view.

- TV annular and RV myocardial tissue Doppler velocities at basal and midventricular level from apical window. Myocardial performance index (MPI) was calculated using the above parameters.

- RV global longitudinal strain from apical RV focused view.

- Tricuspid regurgitation severity and jet velocity from apical window.

- RV dimensions at basal and midlevels from RV apical $\mathrm{RV}$ focused view.

- RV outflow tract dimension from parasternal RV inflow-outflow view.

- Pulmonary artery acceleration time (PAAT) from parasternal RV inflow outflow views. Heart rate (HR) correction was done by dividing PAAT with $\mathrm{HR}$ at the time of measurement to calculate HR corrected PAAT (HRcPAT).

These measurements were performed in the preoperative period in all patients followed by repeat assessments on PODs 2, 7, and 30. An average of three readings was obtained for all the parameters.

\section{Statistical Analysis}

Continuous variables are expressed as mean + standard deviation (SD) and categorical data expressed as numbers and percentages. Serial echocardiographic parameters were compared using repeated measures analysis of variance. A p-value $<0.05$ was considered significant. Intraobserver and interobserver variability in measurements of RV functions (RV FAC, RV MPI, RV strain, and HRcPAT) was assessed in 20 randomly selected subjects offline by an independent observer. Statistical analysis was performed using Statistical Package for the Social Sciences software (IBM SPSS Statistics 20, Chicago, Illinois).

\section{RESULTS}

A total of 32 patients undergoing elective lung surgery (either lobectomy or pneumonectomy) were enrolled in this study. Five patients were excluded due to exclusion criteria and one was lost to follow-up. There was no operative mortality. Table 1 shows the baseline demographics and clinical characteristics of the patients.

Table 1: Baseline and clinical characteristics

\begin{tabular}{ll}
\hline Parameters & Mean $\pm S D(n=26)$ \\
\hline Age (years) & $38.5 \pm 11.3$ \\
Sex & \\
Male & $5(19 \%)$ \\
Female & $21(81 \%)$ \\
BMl & $20.9 \pm 3.5$ \\
$\mathrm{Hb}(\mathrm{mg} / \mathrm{dL})$ & $11.9 \pm 1.4$ \\
Creatinine $(\mathrm{mg} / \mathrm{dL})$ & $0.69 \pm 0.24$
\end{tabular}

PFT

Preoperative $\mathrm{SpO}_{2}(\%) \quad 96 \pm 1.5$

Preoperative MAP $(\mathrm{mm} \mathrm{Hg}) \quad 75 \pm 8.7$

Preoperative $\mathrm{PaO}_{2} / \mathrm{FiO}_{2}(\mathrm{~mm} \mathrm{Hg}) \quad 380 \pm 88$

Preoperative FEV1 (L/min) $\quad 2.6 \pm 0.64$

PPO FEV1 (\%) $\quad 76 \pm 6$

Preoperative FEV1/FVC $\quad 90.4 \pm 14.6$

Intraoperative fluids

Crystalloid $(\mathrm{mL}) \quad 454 \pm 124$

Colloid $(\mathrm{mL}) \quad 423 \pm 91$

Blood $(\mathrm{mL}) \quad 127 \pm 132$

Data are given as means \pm SD. BMI: Body mass index; $\mathrm{Hb}$ : Hemoglobin; $\mathrm{SpO}_{2}$ : Peripheral oxygen saturation; $\mathrm{PaO}_{2}$ : Arterial partial pressure of oxygen; $\mathrm{FiO}_{2}$ : Fraction of inspired oxygen; PPO FEV1: Predicted postoperative FEV1; PFT: Pulmonary function test 


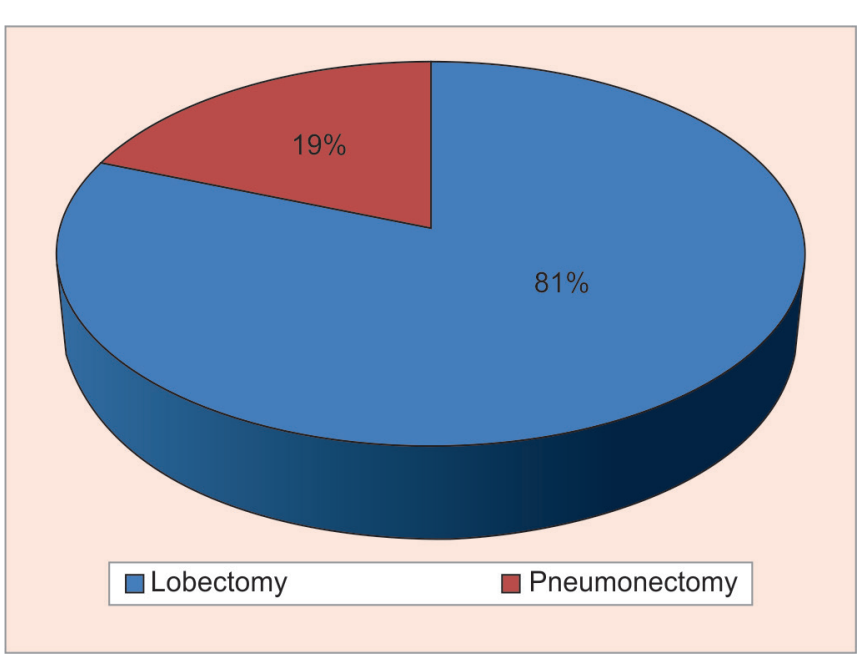

Graph 1: Type of surgery

None of the patients were receiving antihypertensive medications (beta blockers, angiotensin-converting enzyme inhibitors, calcium channel blockers, or diuretics) or required afterload support in terms of vasopressors. No patient developed arrhythmia in the postoperative period. Graph 1 depicts the type of surgeries performed. Left- and right-sided lung resections were performed in 38 and $62 \%$ of the patients respectively.

Table 2 summarizes the echocardiographic parameters of right and LV measured preoperatively and on PODs 2, 7, and 30. None of the patients had RV dilation or dysfunction on preoperative echocardiogram. The RV function decreased significantly on POD 2, which improved slightly thereafter. Postoperatively, mean tricuspid jet velocity (TJV) was $2.6 \pm 0.4 \mathrm{~m} / \mathrm{s}$. Of these patients, $21(42 \%)$ had a TJV of $\geq 2.8 \mathrm{~m} / \mathrm{s}$. Only two patients $(3 \%$, both right-sided lobectomy) had TJV of $\geq 3.4 \mathrm{~m} / \mathrm{s}$. Fifteen patients $(29 \%, 10$ right-sided and 5 left-sided resection) had an RV systolic pressure (RVSP) of $\geq 40 \mathrm{~mm} \mathrm{Hg}$. Preoperative values corresponding to biventricular function were within normal limits in the cohort as well as in the two patient subgroups (Table 3). Graph 2 reveals the trend in RV MPI, RV strain; RV FAC, and HRcPAT. We also compared the measurements according to the extent of resection and side of resection (Table 3). There was no difference between any of the groups. Bland Altman analysis done to see inter- and intraobserver variability (Graphs 3 and 4 respectively) showed $<10 \%$ bias.

\section{DISCUSSION}

The effect of pulmonary resection upon the function of the RV remains unclear. In addition to the complex geometry of the RV, its interaction with the LV makes RV evaluation challenging. The importance of RV function after pulmonary resection has been evaluated previously with techniques, such as invasive hemodynamic measurements and routine echocardiography. $5,9,10$ Some authors have reported a slight postoperative decrease in RV function. ${ }^{1,2,5,11}$ Doppler measurements of RV filling velocities suggest significant dilatation and dysfunction of the RV in the early postoperative period.

Table 2: Echocardiographic parameters

\begin{tabular}{|c|c|c|c|c|c|c|}
\hline Parameters & Preoperative $(n=26)$ & POD $2(n=26)$ & $P O D 7(n=26)$ & POD $30(n=21)$ & $f$-value & p-value \\
\hline \multicolumn{7}{|l|}{ Right ventricle } \\
\hline RV strain (\%) & $-19.2 \pm 4.5$ & $-15.1 \pm 3.7$ & $-17.1 \pm 3.2$ & $-16.8 \pm 7.0$ & 2.79 & 0.045 \\
\hline RV FAC (\%) & $42.6 \pm 12.7$ & $33.7 \pm 10.4$ & $46.6 \pm 8.8$ & $39 \pm 9.1$ & 5.18 & 0.002 \\
\hline RV MPI & $0.35 \pm 0.1$ & $0.44 \pm 0.1$ & $0.32 \pm 0.1$ & $0.37 \pm 0.1$ & 7.27 & $<0.0001$ \\
\hline RVSP (mm Hg) & $21 \pm 16$ & $41 \pm 20$ & $18 \pm 6$ & $27 \pm 14$ & 9.13 & $<0.0001$ \\
\hline TR grade & 0 & 2 & 1 & 0 & 11.5 & $<0.0001$ \\
\hline TV S' (cm/sec) & $13.4 \pm 3.8$ & $10.2 \pm 3.8$ & $12 \pm 4.3$ & $13.3 \pm 4$ & 3.8 & 0.013 \\
\hline PAAT (msec) & $116 \pm 17$ & $91 \pm 12$ & $114 \pm 12$ & $107 \pm 11$ & 17.9 & $<0.0001$ \\
\hline TV E (cm/sec) & $59.2 \pm 16.7$ & $54.6 \pm 16.5$ & $57.3 \pm 13.7$ & $62.5 \pm 20.9$ & 0.89 & 0.450 \\
\hline IVC diameter (mm) & $14 \pm 2.8$ & $19 \pm 3$ & $12 \pm 2.2$ & $15 \pm 2.6$ & 23.39 & $<0.0001$ \\
\hline IVC collapsibility (\%) & $28.6 \pm 9.5$ & $20.9 \pm 9.7$ & $31.8 \pm 10.7$ & $26.6 \pm 9.4$ & 4.46 & 0.006 \\
\hline TV E/A & $0.96 \pm 0.4$ & $4.73 \pm 1.3 ?$ & $2.02 \pm 1.4$ & $4.43 \pm 1.5$ & 40.81 & $<0.0001$ \\
\hline HRcPAT (msec) & $139 \pm 24$ & $111 \pm 21$ & $121 \pm 11$ & $121 \pm 14$ & 9.64 & $<0.0001$ \\
\hline \multicolumn{7}{|l|}{ Left ventricle } \\
\hline GLS (\%) & $-17 \pm 3.4$ & $-16 \pm 2.3$ & $-16 \pm 3.0$ & $-17 \pm 2.4$ & 0.604 & 0.614 \\
\hline LV MPI & $0.30 \pm 0.1$ & $0.34 \pm 0.1$ & $0.26 \pm 0.0$ & $0.30 \pm 0.1$ & 3.66 & 0.016 \\
\hline LVEF (\%) & $56 \pm 7$ & $52 \pm 7.5$ & $56 \pm 4$ & $56 \pm 6$ & 2.59 & 0.058 \\
\hline MV lateral S (cm/sec) & $14.2 \pm 2.1$ & $12.8 \pm 2.3$ & $15.3 \pm 2.2$ & $14.4 \pm 2.2$ & 4.47 & 0.006 \\
\hline MV medial S (cm/sec) & $11.2 \pm 2.5$ & $9.2 \pm 2.5$ & $11.6 \pm 2.5$ & $10.7 \pm 2.6$ & 3.81 & 0.013 \\
\hline
\end{tabular}

Data are expressed as mean \pm SD except TR grade, which is expressed as median; TR: Tricuspid regurgitation; S: Systolic tissue velocity; E/A: Transvalvular early and late diastolic blood flow velocity; IVC: Inferior venacava; GLS: Global longitudinal strain; MV: Mitral valve 


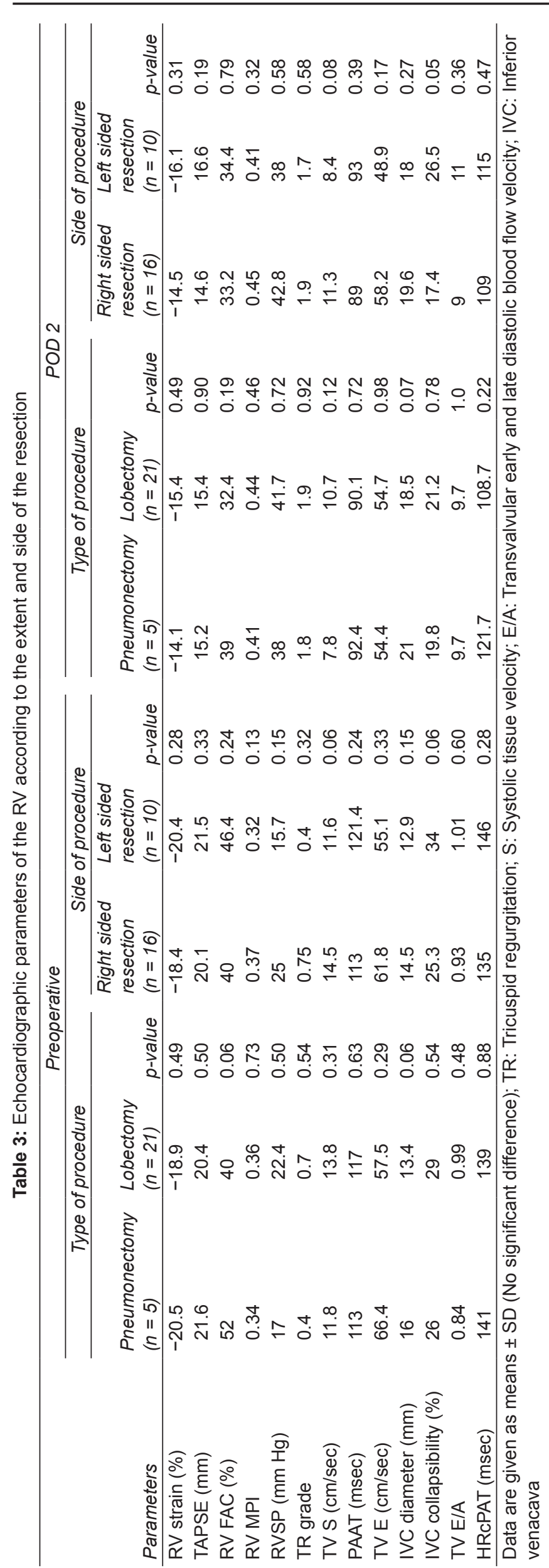

Kumbasar et $\mathrm{al}^{12}$ showed decrease in peak velocity of early diastolic filling I and peak velocity of late diastolic filling (A), but there was no significant change in peak systolic velocity $\left(S^{\prime}\right)$. In contrast, we found a significant fall in $S^{\prime}$ on POD 2, which gradually improved over 30 days. However, TDI values are significantly affected by ventricular volume status, atrial pressures, and rate of myocardial relaxation; therefore, these measures have some limitations on evaluation of systolic and diastolic functions. ${ }^{13,14}$ Similar to other studies, systolic function showed a slight, yet statistically significant decrease in RV function (determined by TAPSE) in the immediate postoperative period, which ameliorates within the first week by compensation. ${ }^{15}$ The extent or side of resection did not have any effect on the function of the RV as measured in our study. This was a major change we observed as compared with previous studies.

Reed et al, ${ }^{9}$ in their study, suggest that RV dysfunction after pulmonary resection is not caused by primary alterations in contractility or immediate changes in afterload. Therefore, normal parameters of RV as assessed by 2D echocardiography may not reveal RV dysfunction due to pneumonectomy/lobectomy. Myocardial strain recorded by $2 \mathrm{D}$ speckle tracking imaging (STI) has been proven to deliver more precise quantifications of regional and global cardiac functions than standard echocardiographic techniques. The peak systolic strain rate correlates well with load independent indices of contractility and, hence, provides valuable information on regional contractile function. Importantly, STI strain data are highly reproducible and analysis is affected by only small intraobserver and interobserver variability. ${ }^{16}$ There are reports evaluating the effects of lung resection on RV by using RV strain. ${ }^{17}$ We demonstrated that in congruence with other parameters, RV strain deteriorated on POD 2 and later improved over one month. However, LV strain did not change significantly postoperatively. An accelerated HR was observed on POD 2 in all patients, which could have affected the strain parameters.

Similar to other studies, RV function significantly decreases on the POD 2, which gradually improved by the 7th POD and stabilized thereafter. ${ }^{1,12,13}$ However, LV function did not differ over the said time period except a slight fall in MV lateral S' on POD 2 (Table 2). No differences in LV function were recorded in terms of extent or side of pulmonary resection.

This study also raises a few questions as to why does the RV function first deteriorate and then improve. What are the putative mechanisms of that deterioration and improvement? Is it a purely mechanical increase in resistance/afterload or are cytokines released during surgery also involved in the changes seen in RV 

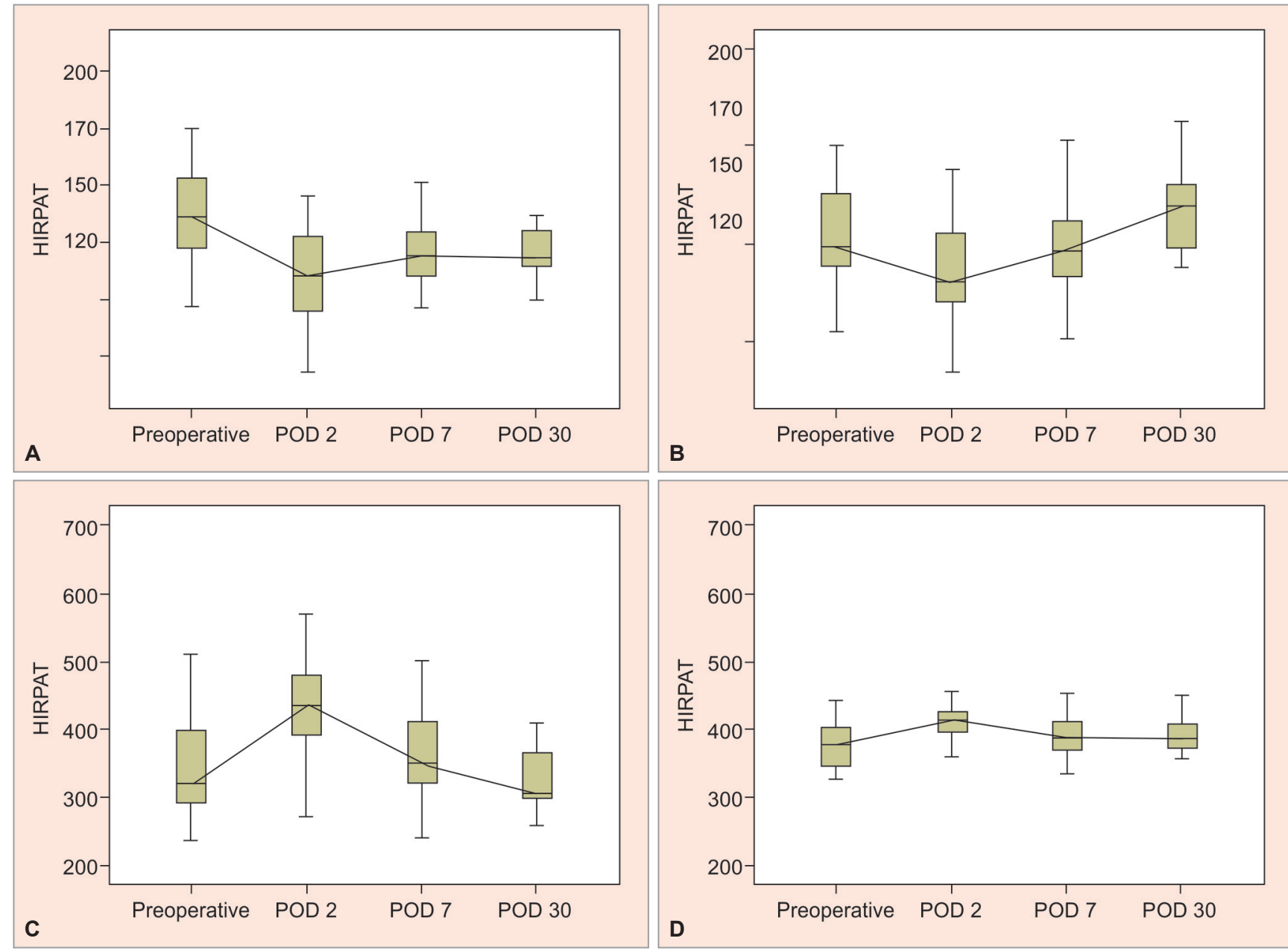

Graphs 2A to D: Box and whisker plot for RV MPI, RV strain, RV FAC, and HRcPAT

FAC/TAPSE/MPI post lung resection? If it is purely mechanical, it should not get better unless the RV subacutely adapts to the reduced pulmonary vascular bed area. If in a lobectomy (smaller change in pulmonary vascular resistance), one sees reduction in RV function, can it be entirely blamed on the increased resistance or there is some other mechanism? Is there a component of systemic inflammatory response syndrome (SIRS)related cardiac dysfunction superimposed on increased $\mathrm{RV}$ afterload that is at play here?

The limitation of this study is that it is based on a small population whose characteristics were heterogeneous. Studies with larger populations and with prolonged follow-up periods can provide more evidence regarding
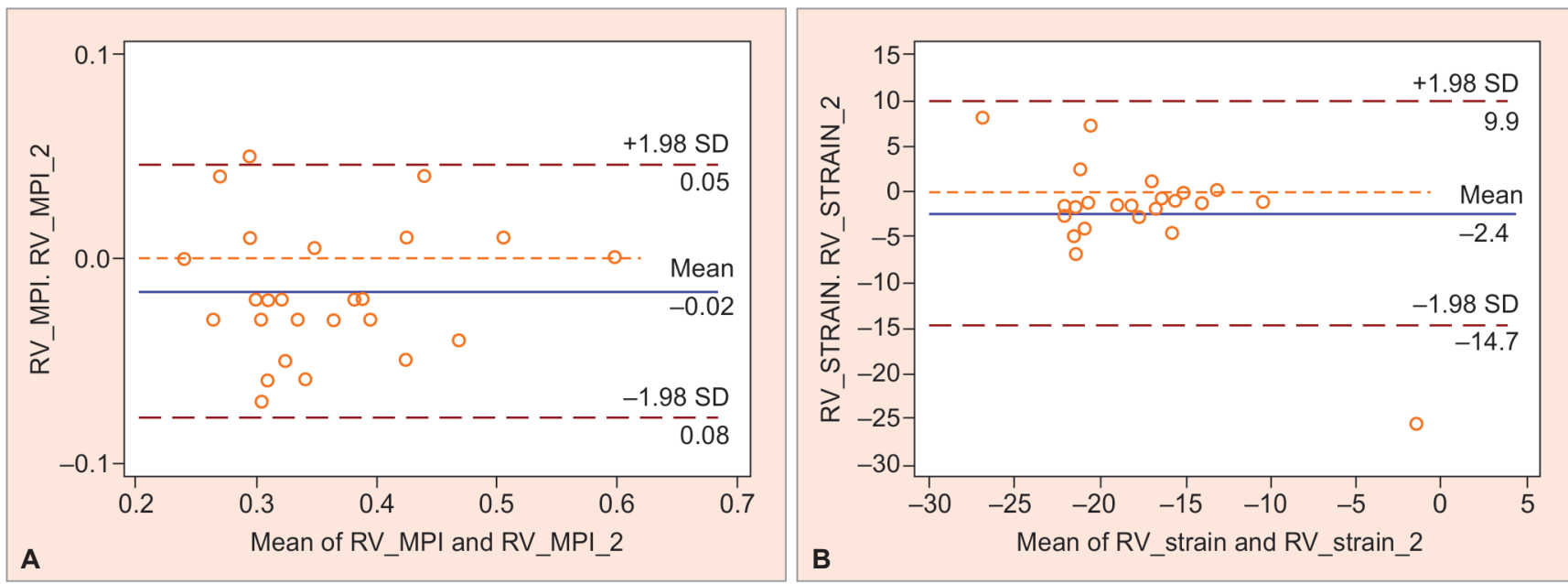

Graphs 3A and B: Interobserver variability for RV MPI, RV strain

(Cont'd...) 
Echocardiographic Evaluation of RV Function
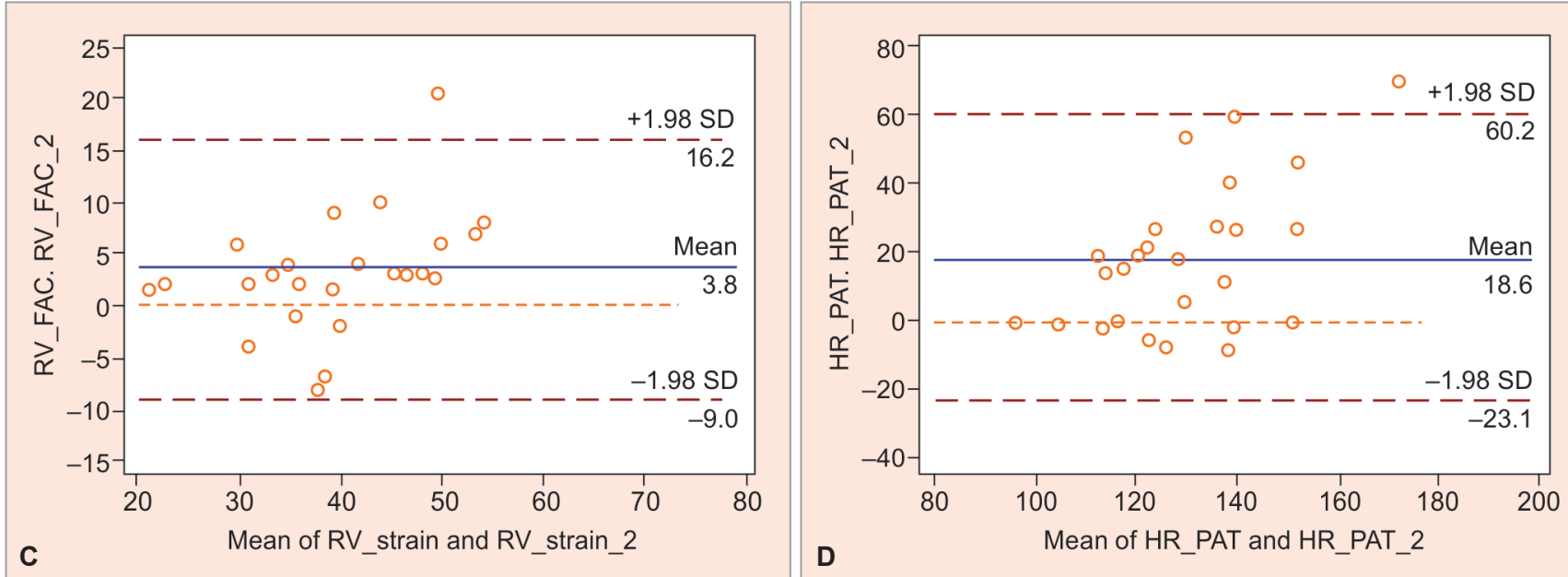

Graphs 3C and D: Interobserver variability for RV FAC, and HRcPAT
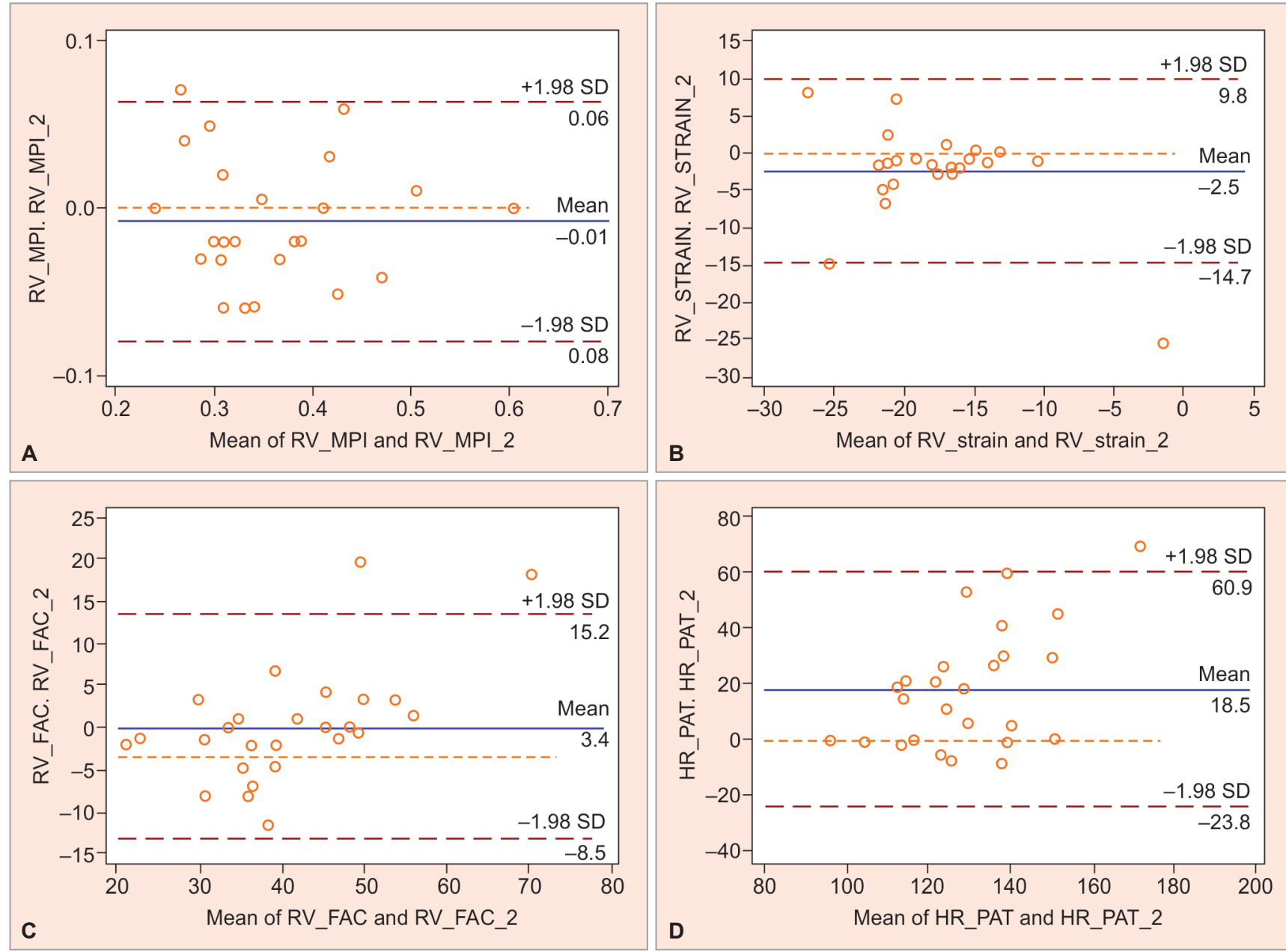

Graphs 4A to D: Intraobserver variability for RV MPI, RV strain, RV FAC, and HRcPAT

postsurgery RV function. There were more lobectomies as compared with pneumonectomy. With lesser degree of change in pulmonary vascular bed and pulmonary vascular resistance in lobectomies as compared with pneumonectomy could have theoretically skewed our result. Also, simultaneous pulmonary function tests can give insight about the mechanism of RV dysfunction in immediate postoperative period and improvement thereafter.

\section{CONCLUSION}

After pulmonary resection, patients develop RV dysfunction in immediate postoperative period (both systolic and diastolic) that compensates over a period of 1 month. 
However, this is accompanied by normal LV function. Early detection of RV deterioration by means of this noninvasive technique could make swift interventional therapy possible, which is an important step toward avoiding future right heart failure.

\section{REFERENCES}

1. Venuta F, Sciomer S, Andreetti C, Anile M, De Giacomo T, Rolla M, Fedele F, Coloni GF. Long-term Doppler echocardiographic evaluation of the right heart after major lung resections. Eur J Cardiothorac Surg 2007 Nov;32(5):787-790.

2. Foroulis CN, Kotoulas CS, Kakouros S, Evangelatos G, Chassapis C, Konstantinou M, Lioulias AG. Study on the late effect of pneumonectomy on right heart pressures using Doppler echocardiography. Eur J Cardiothorac Surg 2004 Sep;26(3):508-514.

3. Kopec SE, Irwin RS, Umali-Torres CB, Balikian JP, Conlan AA. The post-pneumonectomy state. Chest 1998 Oct;114(4): 1158-1184.

4. Harrison RW, Adams WE, Beuhler W, Long ET. Effects of acute and chronic reduction of lung volumes on cardiopulmonary reserve. AMA Arch Surg 1957 Oct;75(4):546-551.

5. Reed CE, Spinale FG, Crawford FA Jr. Effect of pulmonary resection on right ventricular function. Ann Thorac Surg 1992 Apr;53(4):578-582.

6. Yilmaz M, Erol MK, Acikel M, Sevimli S, Apn N. Pulsed Doppler tissue imaging can help to identify patients with right ventricular infarction. Heart Vessels 2003 Jul;18(3):112-116.

7. Hasegawa H, Little WC, Ohno M, Brucks S, Morimoto A, Cheng HJ, Cheng CP. Diastolic mitral annular velocity during the development of heart failure. J Am Coll Cardiol 2003 May;41(9):1590-1597.

8. Sohn DW, Chai IH, Lee DJ, Kim HC, Kim HS, Oh BH, Lee MM, Park YB, Choi YS, Seo JD, et al. Assessment of mitral annulus velocity by Doppler tissue imaging in the evaluation of left ventricular diastolic function. J Am Coll Cardiol 1997 Aug;30(2):474-480.
9. Reed CE, Dorman BH, Spinale FG. Mechanisms of right ventricular dysfunction after pulmonary resection. Ann Thorac Surg 1996 Jul;62(1):225-231.

10. Kowalewski J, Brocki M, Dryjanski T, Kapron K, BarcikowskiS. Right ventricular morphology and function after pulmonary resection. Eur J Cardio Thorac Surg 1999 Apr;15(4): 444-448.

11. Lewis JW Jr, Bastanfar M, Gabriel F, Mascha E. Right heart function and prediction of respiratory morbidity in patients undergoing pneumonectomy with moderately severe cardiopulmonary dysfunction. J Thorac Cardiovasc Surg 1994 Jul;108(1):169-175.

12. Kumbasar U, Kavuksa HS, cardiographic evaluation of the right heart after major lung resections. Eur J Cardiothorac Surg 2007;004; pneumonectomy with moderately se Jan;43(6):971-975.

13. Choong CY, Herrmann HC, Weyman AE, Fifer MA. Preload dependence of Doppler-derived indexes of left ventricular diastolic function in humans. J Am Coll Cardiol 1987 Oct;10(4):800-808.

14. Kuo LC, Quinones MA, Rokey R, Sartori M, Abinader EG, Zoghbi WA. Quantification of atrial contribution to left ventricular filling by pulsed Doppler echocardiography and the effect of age in normal and diseased hearts. Am J Cardiol 1987 May;59(12):1174-1178.

15. Andaluz-Ojeda D, Gandía F, DuqueJL, Gómez I, DeGregorio B, San Román JA, Villacorta E. Biomarkers and echocardiography in the postoperative course of pulmonary resection surgery. Open J Thorac Surg 2011 Sep;1(1):1-8.

16. Becker M, Bilke E, Kuhl H, Katoh M, Kramann R, Franke A, Bücker A, Hanrath P, Hoffmann R. Analysis of myocardial deformation based on pixel tracking in two dimensional echocardiographic images enables quantitative assessment of regional left ventricular function. Heart 2006 Aug;92(8): 1102-1108.

17. Wang Z, Yuan J, Chu W, Kou Y, Zhang X. Evaluation of left and right ventricular myocardial function after lung resection using speckle tracking echocardiography. Medicine (Baltimore) 2016 Aug;95(31):e4290. 